\title{
Ovarian cancer stem-like cells elicit the polarization of M2 macrophages
}

\author{
QING ZHANG ${ }^{1}$, DA-JUN CAI ${ }^{1}$ and $\mathrm{BIN} \mathrm{LI}^{2}$ \\ ${ }^{1}$ Department of Gynecology, The Second Affiliated Hospital of Zhengzhou University, Zhengzhou, Henan 450014; \\ ${ }^{2}$ Department of Gynecology, The First Affiliated Hospital of Zhengzhou University, Zhengzhou, Henan 450052, P.R. China
}

Received April 18, 2014; Accepted December 19, 2014

DOI: $10.3892 / \mathrm{mmr} .2015 .3323$

\begin{abstract}
Ovarian cancer is a life-threatening disease in females worldwide. The polarization of macrophages is crucial in oncogenesis and the development of ovarian cancer. Increasing evidence has supported the correlation between ovarian cancer stem-like cells (OCSCs) and macrophages, however, whether OCSCs can affect the polarization of macrophages and the underlying mechanisms involved remain to be elucidated. To examine the interplay between OCSCs and macrophages, a co-culture system was used to detect the effect of OCSCs on macrophage polarization. The expression of cluster of differentiation 206+ and the secretion of interleukin-10 were significantly increased and the production of tumor necrosis factor- $\alpha$ was suppressed, confirming macrophage polarization to M2 macrophages. Further investigation of the macrophages in a Transwell culture system with OCSCs revealed polarization to the M2 macrophages to a similar extent, indicating that the cytokines of the OCSCs, rather than direct cell-cell contact, are important for the polarization of M2 macrophages. Furthermore, the expression levels of chemokine (C-C motif) ligand (CCL)2, cyclooxygenase (COX)-2 and prostaglandin E2 (PGE2) were increased in the Transwell system and the inhibition of COX-2, but not CCL2, significantly decreased the polarization of the M2 macrophages. In addition, mechanistic analysis revealed the importance of the COX-2/PGE2 pathway in OCSCs to activate Janus kinase (JAK) signaling in macrophages to elicit M2 polarization. These findings provided the first evidence, to the best of our knowledge, that OCSCs are capable of altering macrophages into the M2 phenotype via the overexpression of COX-2 and the increased production of PGE2 cytokines and that the JAK signaling pathway in macrophages is important for this
\end{abstract}

Correspondence to: Dr Bin Li, Department of Gynecology, The First Affiliated Hospital of Zhengzhou University, 1 Jianshe East Road, Zhengzhou, Henan 450052, P.R. China

E-mail: zhangqinghenan@163.com

Key words: ovarian cancer stem-like cells, M2 macrophages, cyclooxygenase-2, Janus kinase alteration. The present study provided evidence supporting possible molecular targets for cancer treatment.

\section{Introduction}

Ovarian cancer is currently the most life-threatening type of malignancy in the female reproductive system and is the fourth leading cause of cancer-associated mortality in females in Europe and the United States (1). Metastasis and recurrence are two of the major difficulties faced in the treatment of ovarian cancer. Ovarian cancer primary disease is treated in $\sim 80 \%$ of patients using surgery and chemotherapy, however, $15 \%$ of patients undergo recrudesce. Patients with advanced-stage ovarian cancer inevitably become refractory to chemotherapy, accelerating the disease progression and rate of mortality (2). The high percentage of recurrence and resistance to current chemotherapeutic agents is a significant burden in the treatment of ovarian cancer. Understanding tumor occurrence, metastasis and the fundamental cause of resistance are key to improving the prognosis of ovarian cancer. Subpopulations of tumor cells with stem cell-like properties have been suggested to function in sustaining the growth of tumor cells, and the inherent drug resistance of these stem-cell-like cells may lead to tumor recurrence following chemotherapy (3).

Over the last decade, the suggestion that tumors are maintained by their own stem cells has been of significant interest to the scientific community. Cancer stem cells have received increasing attention, and the existence of cancer stem cells has been observed in several types of tumor (4). Ovarian cancer stem-like cells (OCSCs) have been successfully isolated with the ability to self-renew and differentiate into non-stem cells, which comprise the bulk of tumor cells (5). Previous investigation has demonstrated that cancer stem cells are important in tumor occurrence, recurrence, metastasis and drug resistance (6). The identification of cancer stem cells provides a good explanation as to why ovarian cancer cells exhibit drug resistance, metastasis and recurrence, and has led to OCSCs being a key focus for ovarian cancer treatment. Evidence has revealed the importance of tumor cells in chemotherapy resistance and tumor progression (7). It is hypothesized that conventional treatments preferentially target rapidly dividing cells and, therefore, OCSCs survive and give rise to recurrent tumors with increased chemoresistance and aggression. In addition to intrinsic genetic and epigenetic signatures in 
tumor cells, tumorigenicity is regulated by extrinsic signals delivered from microenvironments or niches, which are composed of stromal fibroblasts, endothelial cells and inflammatory cells $(8,9)$. In addition, accumulating evidence has revealed the importance of tumor-associated macrophages in tumor progression and metastasis (10) and reports have confirmed that OCSCs are closely associated with their microenvironment and that cancer stem cells are regulated by their microenvironment (11). Additional evidence has demonstrated the regulatory effect of cancer stem cells on the polarization of macrophages in the tumor microenvironment $(12,13)$.

In different microenvironments, macrophages can be polarized to assume different phenotypes and exhibit different functions (14). The tumor microenvironment, providing the required matrix for tumor development, is important for the aggressive behavior of malignant solid tumors (15). Among the cell types associated with the tumor microenvironment, including T cells, B cells, natural killer cells and macrophages, macrophages have the most significant effect on tumor progression $(16,17)$. The polarization of macrophages in the tumor microenvironment is important for tumor progression, and the tumor microenvironment often induces an inflammatory response, recruiting a large number of macrophages (18). The phenotypic profile of macrophages usually includes M1 and M2 phenotypes in the tumor microenvironment. M1 macrophages are generally considered pro-inflammatory and are characterized by the release of reactive oxygen species, nitric oxide, peroxisome proliferator-activated receptor (PPAR)- $\alpha$ and tumor necrosis factor (TNF)- $\alpha$. By contrast, M2 macrophages are considered anti-inflammatory and are characterized by the production of transforming growth factor (TGF)- $\beta$, interleukin (IL)-10, chininase-3-like protein 3 (YM-1) and arginase-1. Tumor-associated macrophages (TAMs), a type of M2-like macrophage, are distributed predominantly in the tumor microenvironment and secrete pro-angiogenic factors, including vascular endothelial growth factor, TGF- $\beta$, PPAR- $\gamma$ and IL-10, which function as anti-inflammatory factors. Previous studies have reported that the density of M2 macrophages is negatively correlated with the survival rate of patients with a variety of tumor types and that an increased density of M2 macrophages accelerates the development of cancer (19). Therefore, it is important to examine the regulatory mechanisms underlying macrophage polarization in the tumor microenvironment. Macrophages can be polarized to assume different phenotypes and exhibit different functions in different microenvironments, and the majority of macrophages in tumor microenvironments are of the M2 type. It has been confirmed that OCSCs with multiple differentiation potentials have the ability to secrete cytokines, which may contribute to the polarization of macrophage cells (20). However, whether OCSCs affect macrophage polarization remains to be elucidated.

\section{Materials and methods}

Cytokines and reagents. Recombinant human IL-4 and chemokine (C-C motif) ligand (CCL)-2, also termed macrophage chemotactic protein-1 (MCP-1) were purchased from PeproTech, Inc (Rocky Hill, NJ, USA). The monoclonal antibodies used in the present study were as follows: Rat anti-human IL-10-phycoerythrin (PE; cat. no. 554498), mouse anti-human TNFa-PE (cat. no. 340512), mouse anti-human cluster of differentiation (CD)206 (cat. no. 551135), rabbit anti-human CCL2 (BD Biosciences, San Jose, CA, USA) and mouse anti-human CD14-PE (cat. no. 325606; BioLegend, Inc, San Diego, CA, USA). AZD1480 was purchased from AstraZeneca Pharmaceuticals, (Waltham, MA, USA) and dissolved in dimethyl sulfoxide (Solarbio, Beijing, China) for in vitro investigations. Rabbit anti-human phosphorylated (p-) Janus kinase (JAK)2 (cat. no. 3776; 1:1,000 dilution), rabbit anti-human JAK2 (cat. no. 3230; 1:1,000 dilution), rabbit anti-human extracellular signal-regulated kinase (ERK; cat. no. 4695; 1:1,000 dilution), rabbit anti-human p-ERK (cat. no. 4377; 1:1,000 dilution), rabbit anti-human Akt (cat. no. 4685; 1:1,000 dilution), rabbit anti-human p-Akt (cat. no. 4058; 1:1,000 dilution) and rabbit anti-human p-signal transducer antibodies and activator of transcription (STAT)3 (Tyr705; cat. no. 9145; 1:1,000 dilution) were obtained from Cell Signaling Technology, Inc. (Danvers, MA, USA). Goat anti-mouse/rabbit immunoglobulin $(\mathrm{Ig}) \mathrm{G}$ secondary antibodies were purchased from Sigma-Aldrich (St. Louis, MO, USA; anti-mouse cat. no. 91618-1G; anti-rabbit cat. no. 43413-1G; 1:1,000 dilution).

Collection of human OCSCs. The present study was approved by the Ethics Committee of The Affiliated Hospital of Zhengzhou University (Zhengzou, China) in accordance with the Declaration of Helsinki. Written informed consent was obtained from all patients/patient's family enrolled in the present study.

Primary ovarian cancer cells in ascites fluid were obtained during ascites diagnostic radical surgery, performed in six female patients (age, 25-60 years) with ovarian cancer at the Affiliated Hospital of Zhengzhou University (Zhengzhou, China), and were categorized as malignant Fédération Internationale de Gynécologie et d'Obstétrique stage III serous adenocarcinoma. The ascites fluid was stored in a refrigerator at $4^{\circ} \mathrm{C}$ for $1-2 \mathrm{~h}$ following collection. The supernatant was discarded and the cells $\left(2 \times 10^{5}\right)$ were resuspended in RPMI-1640 medium (Invitrogen Life Technologies, Carlsbad, $\mathrm{CA}, \mathrm{USA})$ and centrifuged at $1,000 \mathrm{x}$ for $10 \mathrm{~min}\left(4^{\circ} \mathrm{C}\right)$ in order to isolate the primary ovarian cells. The resulting single tumor cells were cultured under stem cell conditions of serum-free Dulbecco's modified Eagle's medium/F12 supplemented (Invitrogen Life Technologies) with $20 \mathrm{ng} / \mathrm{ml}$ human recombinant epidermal growth factor (EGF; Invitrogen Life Technologies), $5 \mu \mathrm{g} / \mathrm{ml}$ insulin (Sigma-Aldrich), $0.4 \%$ bovine serum albumin (BSA; Sigma-Aldrich), $10 \mathrm{ng} / \mathrm{ml}$ basic fibroblast growth factor (bFGF; Invitrogen Life Technologies), $1 \%$ penicillin/streptomycin (Sigma-Aldrich) and 1\% fungizone (Sigma-Aldrich). The samples were incubated at $37^{\circ} \mathrm{C}$ for 7 days and subsequently split and organized into cell clusters. The cells were analyzed within 7 days by flow cytometry, reverse transcription quantitative polymerase chain reaction (RT-qPCR) and western blotting.

Preparation of human macrophages. Human dendritic cells were prepared, according to the method previously described by Patterson (21), with minor modifications (22). Briefly, peripheral blood monocytes (PBMCs) from seven female healthy 
donors (ages, 25-60 years) were isolated from the whole blood (50 $\mathrm{ml}$ samples obtained by venipuncture) by Ficoll-Hypaque gradient centrifugation (2,000 x g then 1,500 x g) (Pharmacia, St. Paul, MN, USA) of buffy coats and washed three times with phosphate-buffered saline (PBS; Invitrogen Life Technologies) prior to being resuspended in complete RPMI-1640 medium containing $10 \%$ fetal calf serum (HyClone, Logan, UT, USA), $2.5 \mathrm{mg} / \mathrm{ml}$ amphotericin B (Sigma-Aldrich), $100 \mathrm{mg} / \mathrm{ml}$ streptomycin sulfate (Sigma-Aldrich) and $100 \mathrm{U} / \mathrm{ml}$ penicillin. The cells $\left(3 \times 10^{6}\right.$ cells $\left./ \mathrm{ml}\right)$ were inoculated into a volume of $25 \mathrm{ml}$ in $250 \mathrm{~cm}^{2}$ plastic tissue culture flasks (Corning, Inc., Corning, $\mathrm{NY}$, USA) for $45 \mathrm{~min}$ at $37^{\circ} \mathrm{C}$ in $5 \% \mathrm{CO}_{2}$ and were then plated at a density of $2 \times 10^{5}$ cells/well into 6-well plates (Corning, Inc.) in duplicate. Rinsing with warm PBS removed the non-adherent cells, and the remaining adherent cells were harvested and cultured in complete RPMI-1640 medium. Human recombinant granulocyte macrophage colony-stimulating factor (800 U/ml; Immunex, Seattle, WA, USA) was added to the culture medium to isolate human macrophages.

Co-culture of macrophages with OCSCs. For the co-culture investigations, $2 \times 10^{5}$ OCSCs were seeded with an equal number of human PBMC-derived macrophages $\left(2 \times 10^{5}\right)$ on day 7 of culture and then cultured for a further 3 days in RPMI-1640 medium. For the Transwell co-culture, cultivation was performed, as described previously (23). Briefly, $0.4 \mu \mathrm{m}$ pore-size Corning Transwell inserts (VWR International, Inc., West Chester, PA, USA) were placed into 6-well plates with $2 \times 10^{5}$ macrophages, initially seeded at the bottom of the chamber and $2 \times 10^{5}$ OCSCs seeded onto the inserts, followed by culture for a further 3 days (24).

Flow cytometry. The macrophages $\left(2 \times 10^{5}\right)$ were stained using anti-human CD14-PE, anti-mouse/human CD206-allophycocyanin (APC)/Cy7 and anti-human E-cadherin-fluorescein isothiocyanate (FITC; panisotype) for $20 \mathrm{~min}$ at $4^{\circ} \mathrm{C}$ to detect the macrophage polarization phenotype. To further detect intracellular cytokines, the macrophages were cultured in the presence of $10 \mu \mathrm{g} / \mathrm{ml}$ Brefeldin A (Sigma-Aldrich) to inhibit the secretion of cytokines (26). The cells $\left(2 \times 10^{5}\right)$ were then incubated with specific antibodies for the two cytokines IL-10 and TNF- $\alpha$, and were analyzed by flow cytometry.

$R T-q P C R$. The total RNA was isolated using TRIzol reagent (Invitrogen Life Technologies) and the optical absorbance ratio at 260/280 nm was measured using a ScanDrop100 nucleic acid analyzer (Analytik, Beijing, China) to determine the RNA content. Total RNA (5 $\mu \mathrm{g})$ was reverse-transcribed into cDNA using M-MLV Reverse Transcriptase (Clontech Laboratories, Inc., Palo Alto, CA, USA). cDNAs were used as templates for qPCR. The qPCR mixture contained $5 \mathrm{ml} \mathrm{SsoFast}{ }^{\mathrm{TM}}$ EvaGreen Supermix (Bio-Rad Laboratories, Inc., Hercules, CA, USA), $1 \mathrm{ml}$ cDNA (dilution, 1:50) and $2 \mathrm{ml}$ each of the forward and reverse primers $(1 \mathrm{mM})$ to a final volume of $10 \mathrm{ml}$. qPCR was performed using the cDNA as the template to amplify the mRNA of CCL-2 and cyclooxygenase (COX)-2, using specific primers synthesized by Sangon Biotech. Co., Ltd. (Shanghai, China). The reaction was performed as follows: $10 \mathrm{~min}$ at $95^{\circ} \mathrm{C}$, followed by 40 cycles of $1 \mathrm{~min}$ at $95^{\circ} \mathrm{C}, 2 \mathrm{~min}$ at $63^{\circ} \mathrm{C}$ and
$1 \mathrm{~min}$ at $72^{\circ} \mathrm{C}$, prior to a final annealing step at $72^{\circ} \mathrm{C}$ for $10 \mathrm{~min}$. The mRNA expression levels of CCL-2 and COX-2 were normalized against human GAPDH using the comparative $\mathrm{Ct}$ method (25). The sequences of the primers were as follows: CCL-2, sense 5'-GTTGACCCGTAAATCTGAAGC-3' and antisense 5'-AAGGCATCACAGTCCGAGTCA-3' and COX-2, sense 5'-CACATCCTCAATACCAGGTCC-3' and antisense 5'-CAGAGATCCAGACTCGCATG-3' (26,27).

Western blotting. Macrophages $\left(2 \times 10^{5}\right)$ in Transwell plates were lysed using $200 \mu \mathrm{l}$ lysis buffer (Invitrogen Life Technologies) containing $20 \mathrm{mmol} / 1 \mathrm{HEPES}, 25 \mathrm{mmol} / 1 \mathrm{MgCl}, 5 \mathrm{mmol} / \mathrm{KCl}$, $0.5 \%(\mathrm{v} / \mathrm{v})$ complete protease inhibitor and Triton X-100. The debris was removed by centrifugation at $12,000 \mathrm{x}$ at $4^{\circ} \mathrm{C}$ for $10 \mathrm{~min}$. Western blotting was performed using the total protein from cell lysate homogenates. Equal concentrations of the protein $(80 \mu \mathrm{g})$ were separated using $8 \%$ precast SDS-PAGE gels (Invitrogen Life Technologies) and electrophoretically transferred onto a polyvinylidene fluoride membrane (Bio-Rad Laboratories, Inc.). The membrane was subsequently probed with a 1:150 dilution of polyclonal primary YM-1 or PPAR $\gamma$ antibodies (BD Transduction Laboratories, San Jose, CA, USA) or a 1:1,000 dilution of mouse anti-human prostaglandin E2 (PGE2; Cell Signaling Technology, Inc.), Akt, JAK or ERK antibodies, at $37^{\circ} \mathrm{C}$ for $2 \mathrm{~h}$. Membranes were then washed in PBST five times and then incubated with goat anti-mouse/ rabbit IgG (1:5,000; Sigma-Aldrich) secondary antibodies. Bands were visualized using an enhanced chemiluminescence western blot detection system (GE Healthcare Life Sciences, Chalfont, UK) was performed. The intensity of the bands were measured using Image J software, version 1.48 (National Institutes of Health, Bethesda, MA, USA). For western blot, GAPDH served as a loading control.

Statistical analysis. The data were analyzed using SPSS 17.0 statistical software (SPSS, Inc., Chicago, IL, USA) and the data are expressed as the mean \pm standard deviation. Methods of least significant difference were applied appropriately to evaluate the differences between various groups. $\mathrm{P}<0.05$ was considered to indicate a statistically significant difference.

\section{Results}

Ovarian cancer stem-like cells convert macrophage polarization into the M2 phenotype. The majority of macrophages in the tumor microenvironment exhibit an M2-like phenotype, which is closely associated with tumor development and progression (28). Previous studies have demonstrated that there is a molecular interaction between stem-like cells and macrophages $(29,30)$, however, whether OCSCs affect macrophage polarization, and the specific underlying molecular mechanisms remain to be elucidated. In the present study, following co-culture of OCSCs at equal cell densities for $72 \mathrm{~h}$, under direct cell-cell contact, the expression of CD206, a specific marker of the M2 phenotype, was significantly increased compared with the control (11.7 vs. 40.6\%; $\mathrm{P}<0.05$; Fig. $1 \mathrm{~A})$. A previous investigation confirmed that macrophages of the M2 phenotype increased the expression of anti-inflammatory cytokines, including IL-10, and inhibited the expression of inflammatory cytokines, including TNF $\alpha$ (30). The present study observed 
A
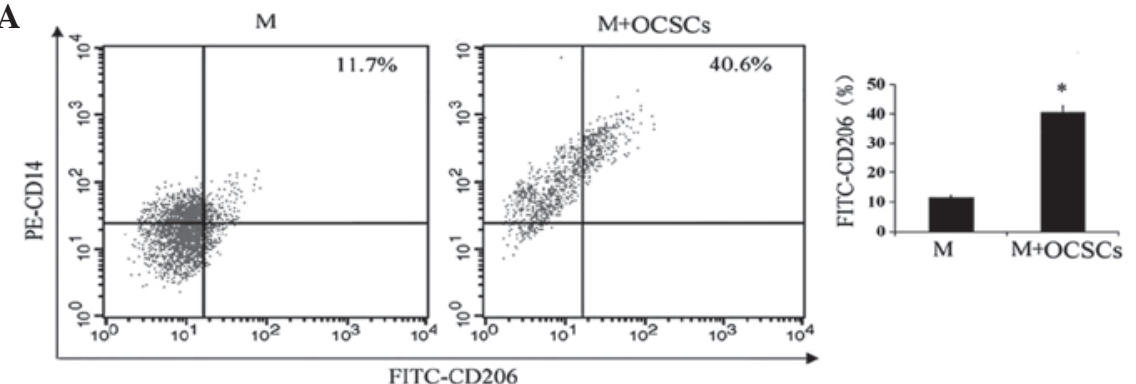

B
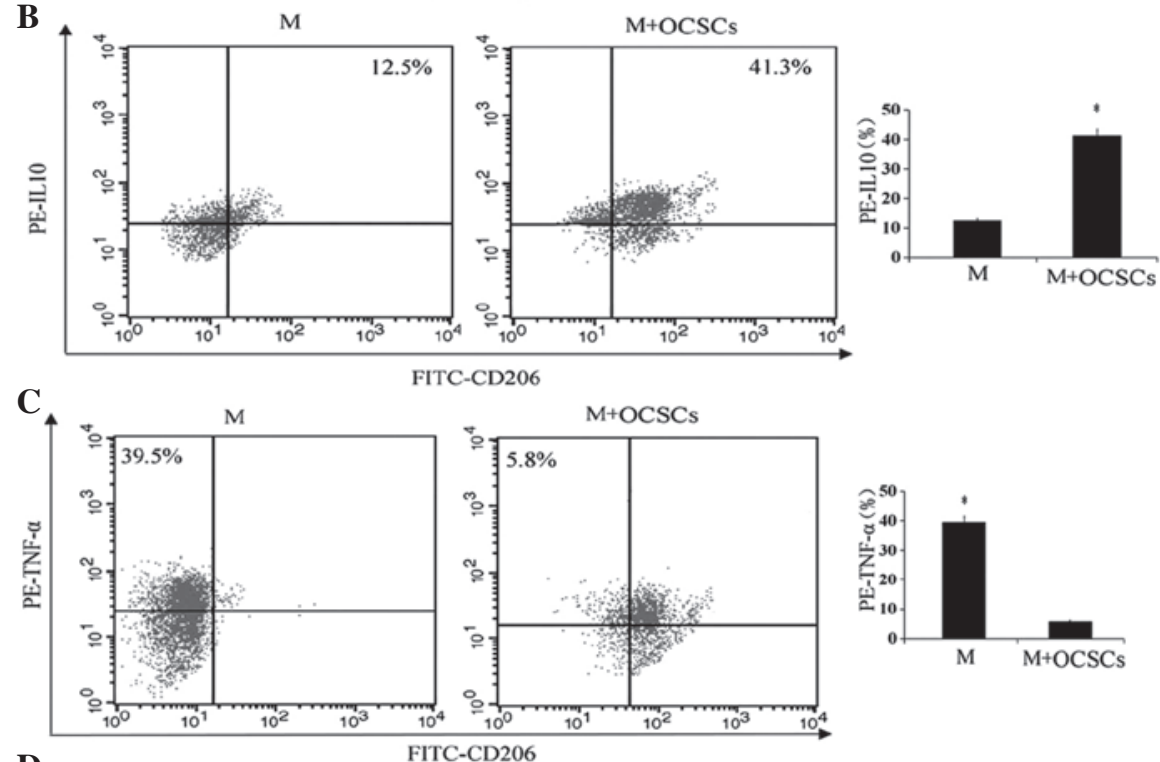

D
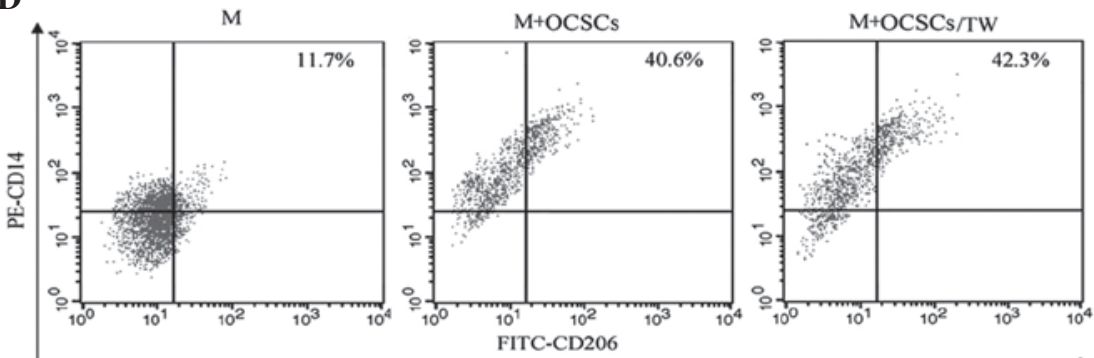

$\mathbf{E}$
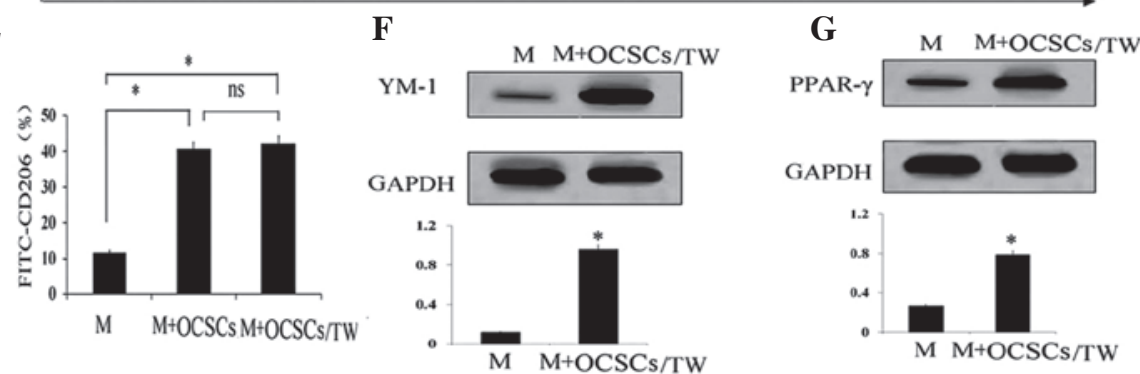

Figure 1. OCSCs promote the polarization of M2 macrophages. Monocytes isolated from PBMCs were seeded into 6-well plates (2x10 $0^{5}$ per well) and cultured in macrophage growth medium for 7 days. An identical number of OCSCs and macrophages were co-cultured for 3 days and the co-cultured cells were collected and immunostained using PE-CD14, FITC-CD206, IL-10 or TNF- $\alpha$ antibodies and analyzed by flow cytometry. The expression levels of (A) CD206, (B) IL-10 and (C) TNF- $\alpha$ were observed in the macrophages and macrophages co-cultured with OCSCs. (D) An identical number of OCSCs and macrophages were co-cultured for 3 days and the co-cultured cells were collected and immunostained using PE-CD14 and FITC-CD206 antibodies, and analyzed by flow cytometry. Expression of CD206 was observed in M, M+OCSCs and M+OCSCs/TW and (E) was quantified. The expression levels of (F) YM-1 and (G) PPAR- $\gamma$ were analyzed by western blotting of M and M+OCSCs/TW. Values are presented as the mean \pm standard deviation. OCSC, ovarian cancer stem-like cell; PBMC, peripheral blood monocyte; CD, cluster of differentiation; FITC, fluorescein isothiocyanate; IL, interleukin; TNF, tumor necrosis factor; YM, chitinase-3-like protein 3; PPAR, peroxisome proliferator-activated receptor; TW, Transwell; M, macrophages cultured alone; M+OCSCs; macrophages co-cultured in direct contact with OCSCs; PE, phycoerythrin; ns, non-signficant.

that co-culture with OCSCs under direct cell-cell contact led to a significant increase in the expression of IL-10 (41.3\%) and a decreased expression of TNF $\alpha(5.8 \%)$ in the macrophages compared with the controls (12.5 and 39.5\%, respectively; Fig. 1B and C). This result indicated that the macrophages with the M2 phenotype and with anti-inflammatory activity 
A

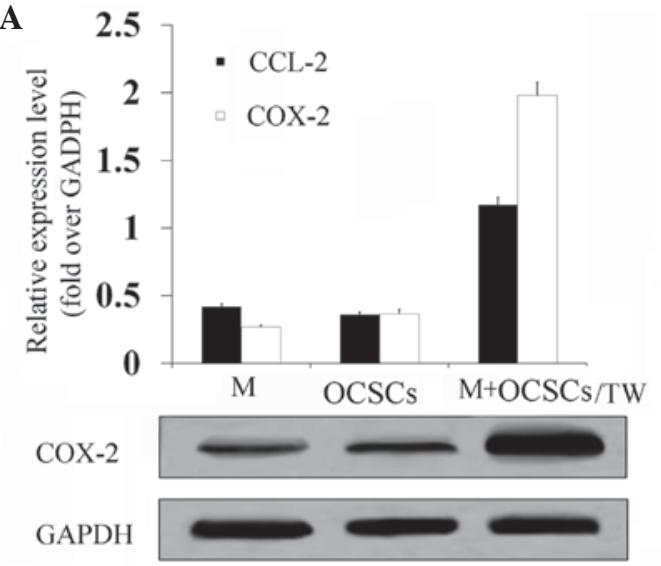

B
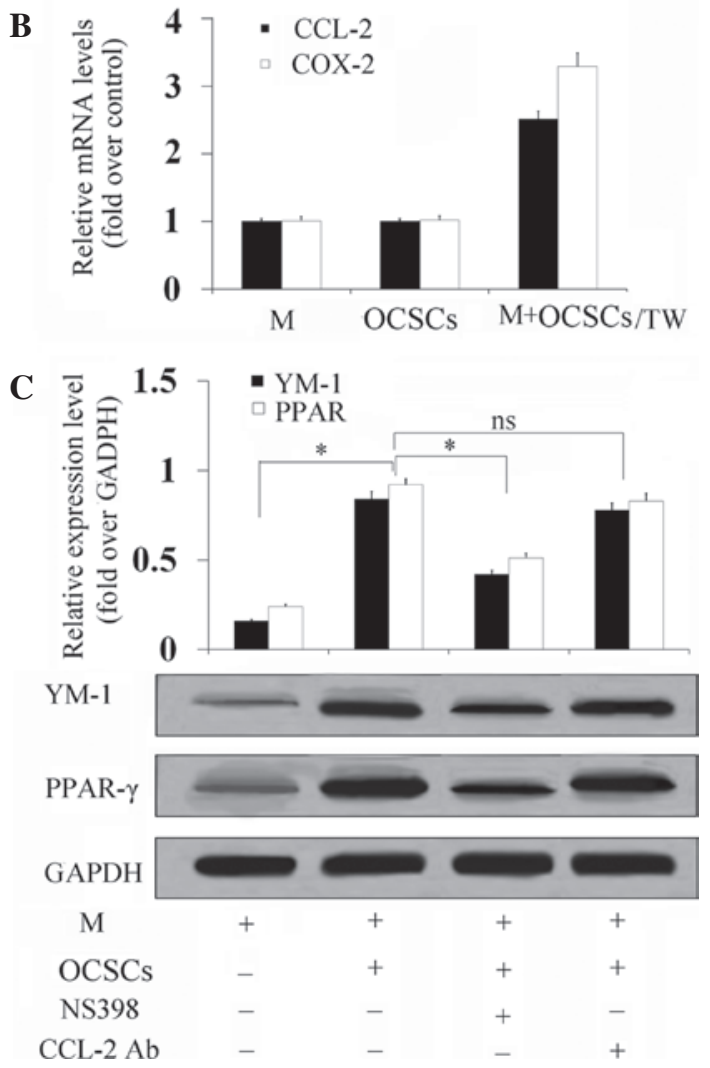

Figure 2. Upregulation of COX-2 in OCSCs is important in converting the macrophage phenotype. (A and B) Expression levels of CCL-2 and COX-2 were evaluated in macrophages cultured alone and in a trans-well system. (C) Expression levels of YM-1 and PPAR- $\gamma$ were analyzed by western blotting in macrophages cultured alone or in a Transwell system treated with the CCL-2 antibody. Values are presented as the mean \pm standard deviation. COX, cyclooxygenase; OCSC, ovarian cancer stem-like cell; CCL, chemokine (C-C motif) ligand 2; PBMC, peripheral blood monocyte; YM chitinase-3-like protein 3; PPAR, peroxisome proliferator-activated receptor; TW, Transwell; M, macrophages; ns, non-significant.

were induced by OCSCs and confirmed that OCSCs had the potential to convert macrophages into the M2 phenotype. To further examine whether the OCSC-induced upregulation of CD206 was dependent on soluble factors and/or direct cell-cell contact, the OCSCs and macrophages were co-cultured in a Transwell system. The results demonstrated that co-culturing of macrophages with OCSCs in Transwells increased the CD206+ macrophage population to a similar extent as under conditions of direct cell-cell contact (40.6 vs. $42.3 \%$; $\mathrm{P}<0.05$; Fig. 1D and E). Additionally, no significant change was detected in the macrophages cultered alone, indicating that the soluble factors secreted by OCSCs, rather than direct cell-cell contact, contributed to macrophage polarization. Changes in macrophages depend predominantly on the specific cytokines expressed, including YM1, YM2 and resistin-like molecule $\alpha 1$, PPAR- $\gamma$ is the cytokine responsible for increasing the expression of the M2 gene (31). The expression levels of YM-1 and PPAR- $\gamma$ are used as specific markers of alternatively activated macrophages, therefore, their expression levels were assessed by western blotting. The results revealed a significant increase in the expression levels of YM-1 and PPAR- $\gamma$ when the macrophages were co-cultured with OCSCs in Transwells, compared with macrophages cultured alone (Fig. 1F and G). This further confirmed that anti-inflammatory M2 macrophages were induced following co-culture with OCSCs.

Upregulation of COX-2 in OCSCs is important in converting the macrophage phenotype. COX-2 is an inducible enzyme involved in prostaglandin biosynthesis and often overexpressed in several epithelial malignancies, including ovarian and breast cancer $(32,33)$. The overexpression of COX-2 increases the motility and invasion of cancer cells in several types of tumor, including ovarian cancer $(34,35)$. The CC chemokine, CCL2, is overexpressed in ovarian cancer tumor microenvironments and is important for the progression of ovarian cancer. COX-2 and CCL2 have been implicated in inducing M2-type macrophage polarization $(36,37)$, therefore, the present study evaluated the expression levels of CCL2 and COX-2 by western blot analysis of the samples obtained from Transwell co-cultures of OCSCs and macrophages. The results demonstrated that the expression levels of CCL2 and COX-2 were increased compared with the culture of either alone (Fig. 2A). Quantitative intensity analysis revealed that the expression of CCL-2 was increased $\sim 3$-fold and the expression of COX-2 was increased $\sim 5$-fold (Fig. $2 \mathrm{~A}$; $\mathrm{P}<0.05$ ). RT-qPCR analysis revealed that the mRNA expression levels of CCL2 and COX-2 were increased in the OCSCs following Transwell co-culture (Fig. 2B). Inhibition of COX-2 significantly decreased the expression levels of YM- 1 and PPAR- $\gamma$, however, no significant difference was detected following the inhibition of CCL-2 using a CCL-2 antibody (Fig. 2C). These findings suggested that COX-2, but not CCL-2, is important for altering the phenotype of tumor-associated macrophages from $\mathrm{M} 1$ to $\mathrm{M} 2$ in OCSC-induced macrophages.

COX-2/PGE pathway is important for converting macrophage phenotype. Previous studies have suggested that the frequently upregulated expression of COX-2 in tumor cells causes increased PGE2 metabolism, which can facilitate tumor cell transformation, proliferation, angiogenesis and infiltration or migration $(38,39)$. Whether the OCSC-mediated conversion of the macrophage M2 phenotype, via increased expression of the COX-2/PGE pathway, is important remains to be elucidated. In the present study, the expression of PGE2 was further evaluated and the results demonstrated that OCSCs co-cultured with macrophages led to a significant increase in the expression of PGE2 (Fig 3A). The addition of the COX-2 inhibitor, NS398, to the OCSC Transwells prevented the increased expression of PGE2 (Fig. 3A). Following treatment with the anti-PGE2 antibody, 
A

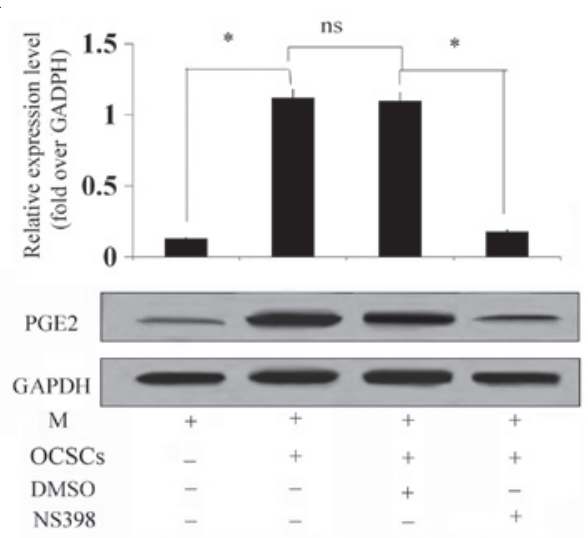

B

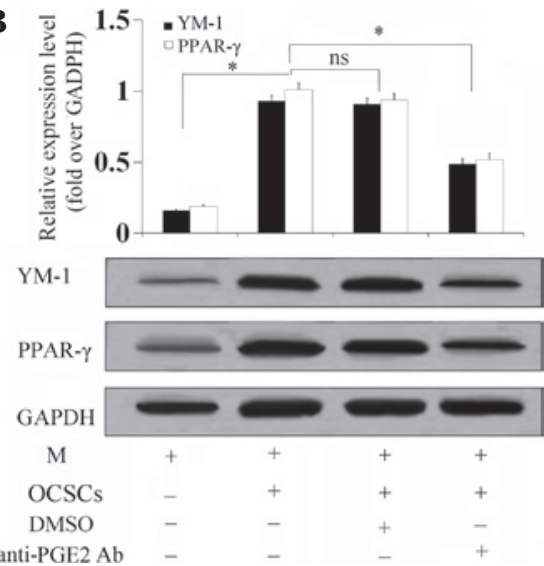

Figure 3. COX-2/PGE pathway is important in converting macrophage phenotype. Macrophages were cultured alone or in a Transwell system and treated with NS398 or DMSO. Expression levels of (A) PGE2, (B) YM-1 and PPAR- $\gamma$ were analyzed by western blotting. Values are presented as the mean \pm standard deviation. OCSC, ovarian cancer stem-like cell; COX, cyclooxygenase; PGE, prostagandin E; YM, chitinase-3-like protein 3; PPAR, peroxisome proliferator-activated receptor; DMSO, dimethyl sulfoxide; $\mathrm{M}$, macrophages.

A

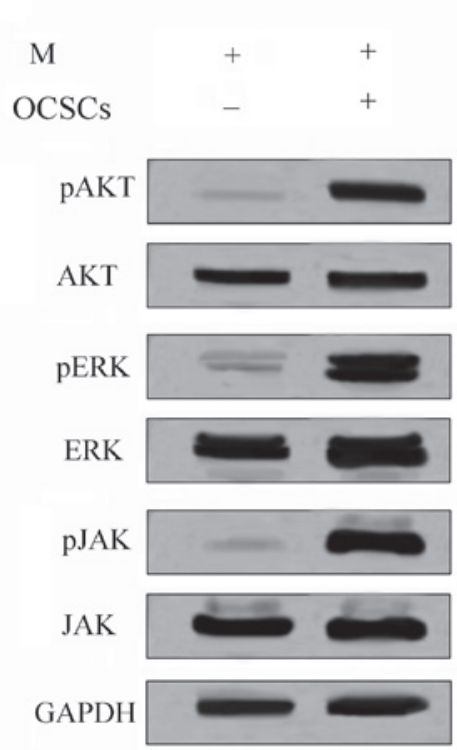

B

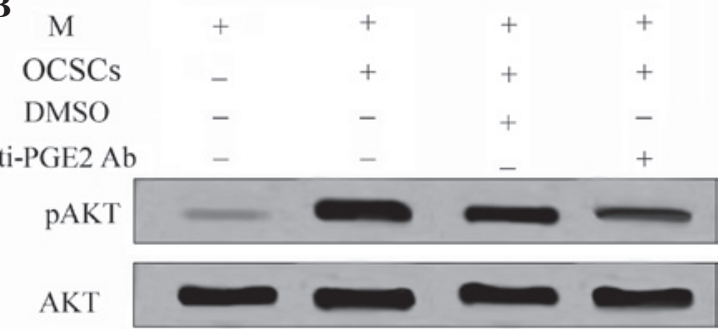

pERK

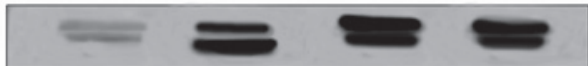

ERK

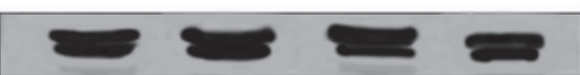

pJAK

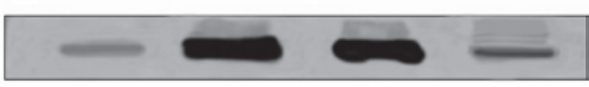

JAK

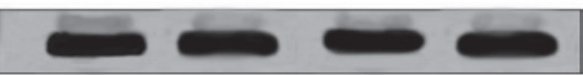

GAPDH

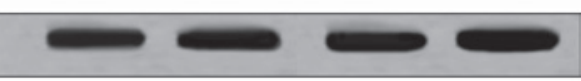

D

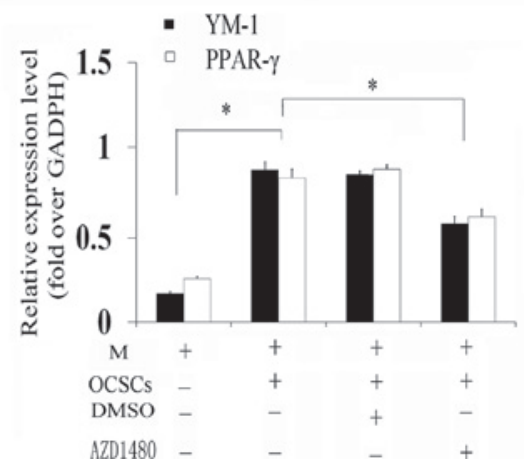

Figure 4. JAK signaling pathway is responsible for converting the macrophage phenotype. (A) Macrophages were cultured alone or in a Transwell system, and the activation of the signaling molecules JAK, ERK and Akt were observed. (B) Macrophages were then cultured alone or in a Transwell system, treated with anti-PGE2 antibodies or DMSO, and the activation of JAK, ERK and Akt were observed. (C and D) Macrophages were cultured alone or in a Transwell system, treated with AZD1480 or DMSO, and the expression levels of YM-1 and PPAR- $\gamma$ were analyzed by western blotting. Values are presented as the mean \pm standard deviation. JAK, janus kinase; OCSC, ovarian cancer stem-like cell; TW, Transwell; DMSO, dimethyl sulfoxide; ERK, extracellular signal-regulated kinase; PGE2, prostaglandin E2; YM, chitinase-3-like protein 3; PPAR, peroxisome proliferator-activated receptor, p, phosphorylated, M, macrophages.

OCSCs exhibited decreased expression levels of YM-1 and PPAR- $\gamma$ (Fig. 3B), indicating that the COX-2/PGE2 pathway is important in altering macrophage polarization.
JAK signaling pathway is responsible for converting the macrophage phenotype. In mammals, the COX-2/PGE2 signaling pathway is propagated by the activation of several 
other proteins and pathways, including the transduction and activation of JAK/STAT and the ERK and phosphatidylinositol 3-kinase (PI3K)-Akt pathways. To investigate the intracellular signaling mechanisms responsible for the altered polarization effect of OCSCs on macrophages, the present study examined the changes in the phosphorylation levels of JAK, ERK and Akt in the macrophages following OCSC stimulation. As shown in Fig. 4A, the phosphorylation of Akt was increased following co-culture with OCSCs. Similarly, co-culturing with OCSCs significantly increased the phosphorylation of ERK and JAK (Fig. 4A).

Furthermore, treatment with the anti-PGE2 antibody abrogated the increased expression of p-JAK, however, no significant effect was observed on the expression levels of p-ERK or Akt (Fig. 4B). In addition, inhibition of JAK with AZD1480 caused a significant decrease in the production of YM-1 and PPAR- $\gamma$ (Fig. 4C and D), suggesting that the JAK signaling pathway exerts important effects in the COX-2/PGE2-induced polarization of M2 macrophages.

\section{Discussion}

Early stage ovarian cancer is difficult to diagnose and is often associated with a poor prognosis when diagnosed at a later stage. OCSCs have become an attractive target for treatment due to their ability to self-renew and differentiate into non-stem cells, which have a decisive role in cancer outcomes (5). Previous studies have demonstrated that the majority of macrophages accumulate in the tumor microenvironment and are important during the onset and progression of ovarian cancer, implying potential targets for anticancer therapy (40). The M1 and M2 macrophage phenotypes are closely involved in inflammation and host defense. It is widely accepted that M2 macrophages are anti-inflammatory and are important in tumor progression (41). Additionally, studies have confirmed that TAM, a distinct M2 polarized population, promote tumor progression and are induced by ovarian cancer cells (42). It has been demonstrated that stem cells potentiate tumor growth through the secretion of soluble signaling molecules (43). Whether OCSCs induce the alteration of macrophages into a tumorigenesis-promoting M2 macrophage phenotype and its underlying mechanisms remain to be elucidated.

The present study demonstrated that cultured OCSCs promoted macrophage differentiation and expression of the M2 macrophage marker, CD206, was increased. Consistent with this result, the expression of the anti-inflammatory cytokine, IL-10, was upregulated, accompanied by downregulation of the inflammatory cytokine, TNF $\alpha$. IL-10 is a cytokine with anti-inflammatory properties and $\mathrm{TNF} \alpha$ predominantly functions in immunity and inflammation. The upregulation of IL-10 and downregulation of TNF $\alpha$ in macrophages may contribute to the evasion of immune mechanisms by limiting the immune response to pathogens (44). Therefore, these results indicated an important role for OCSCs in the polarization of macrophages into the M2 phenotype. Combined with previous studies demonstrating that M2 macrophages promote tumorigenesis and tumor evasion of the immune system (40), it was considered that OCSCs may promote tumorigenesis, metastasis and anti-inflammatory mechanisms via the regulation of macrophage polarization $(40,45)$.
CCL2, also termed MCP-1, is accepted as having a direct role in inflammation, tumorigenesis and chemotaxis. The mass production of CCL2 in the ovarian tumor microenvironment has multiple effects, which have been confirmed in several tumor types and host cells (46). It is understood that CCL2 is involved in recruiting M1 macrophages into the microenvironment of a tumor and their conversion into M2 macrophages (47). The expression of MCP-1 was significantly associated with the accumulation of TAM, a key agonist, which attracts macrophages to tumors $(48,49)$. In addition, it is accepted that the overexpression of COX-2 is important for affecting the development of cancer (50). The deregulation of COX-2 appears to affect tumorigenesis via a number of distinct mechanisms, including promoting tumor maintenance and progression, encouraging metastatic spread and possibly being involved in tumor initiation. To investigate whether CCL2 and MCP-1 function in OCSC-induced macrophage polarization, the present study analyzed the expression levels of CCL2 and COX-2 in OCSCs. Western blot analysis revealed significantly increased expression levels of CCL2 and COX-2. A previous study demonstrated that the inhibition of COX-2 alters the phenotype of tumor-associated macrophages from M2 to M1, suggesting a close association between COX-2 and macrophage polarization (51). To further elucidate the underlying mechanism, the expression of CCL2 and COX-2 were inhibited using specific antibodies and inhibitors. The results demonstrated that the COX-2 inhibitors significantly abrogated the increased expression of YM-1 and PPAR- $\gamma$, however, the CCL2 antibody had no significant effect on the increased expression level of YM-1 and PPAR- $\gamma$. These findings confirmed that OCSCs may trigger the proliferation of M2 macrophages by upregulating COX-2.

The increased levels of PGE2 in the blood of patients with cancer exert inhibitory effects on cellular immunity and promote tumorigenesis (52). A previous study reported that alterations of intracellular PGE2 catabolism induces M2-polarized macrophages (53). The tumor-derived COX-2/PGE2 signaling pathway was demonstrated to inhibit M1 macrophages and induce macrophage polarization to the M2 phenotype (54). To further investigate the underlying molecular mechanisms of the OCSC-induced polarization of M2 macrophages, the present study assessed the expression of PGE2. The expression of PGE2 was increased in the co-culture system and the inhibition of $\mathrm{COX}-2$ decreased the expression of PGE2, suggesting that OCSCs-derived COX-2/PGE2 is important in inhibiting M1 macrophages and inducing M2 macrophages. Multiple signaling pathways have been implicated in the activation of the M2 macrophages, including phosphatidylinositol 3-kinase (PI3K)/Akt, JAK2/STAT3 and mitogen activated protein kinase/ERK $(55,56)$. The present study investigated the underlying signaling pathway of COX-2/PGE2-mediated M2 macrophage polarization. The expression levels of Akt, JAK and ERK were increased to similar extents. The inhibition of PGE2 significantly inhibited the expression of JAK, however no significant effect was observed on the expression of ERK and Akt, indicating that JAK may predominantly function in COX-2/PGE2-mediated M2 macrophage polarization. Additionally, anti-PGE2 antibodies markedly reduced the activation of JAK signaling. This suggested that the PGE2 secreted by OCSCs may affect the 
JAK signaling in macrophages and induce macrophage polarization, while the increased expression of ERK and Akt may correlate with other metabolic pathways and were not further examined in the present study. In addition, the inhibition of JAK by AZD1480 demonstrated a significant reduction in the production of YM- 1 and PPAR- $\gamma$, indicating the importance of the JAK signaling pathway in the OCSC-induced polarization of M2 macrophages.

In conclusion, the present study demonstrated, for the first time to the best of our knowledge, that human-derived OCSCs can switch the differentiation of human macrophages into an anti-inflammatory M2 phenotype via the activation of the COX-2/PGE2 pathway and this further activated the JAK signaling pathway. These findings suggested novel directions for further investigation and potential therapeutic targets for the effective prevention and treatment of ovarian cancer.

\section{References}

1. Hamilton W, Peters TJ, Bankhead C and Sharp D: Risk of ovarian cancer in women with symptoms in primary care: Population based case-control study. BMJ 339: 2998-3005, 2009.

2. Torri V, Harper PG, Colombo N, Sandercock J and Parmar MK: Paclitaxel and cisplatin in ovarian cancer. J Clin Oncol 19: 2349-2351, 2000

3. Dean M: ABC transporters, drug resistance, and cancer stem cells. J Mammary Gland Biol Neoplasia 14: 3-9, 2009.

4. Clarke MF and Fuller M: Stem cells and cancer: two faces of eve. Cell 124: 1111-1115, 2006.

5. Alvero AB, Chen R and Fu HH: Molecular phenotyping of human ovarian cancer stem cells unravel the mechanisms for repair and chemo-resistance. Cell Cycle 8: 158-166, 2009.

6. Dean M, Fojo T and Bates S: Tumour stem cells and drug resistance. Nat Rev Cancer 5: 275-284, 2005.

7. Johnstone RW, Ruefli AA and Lowe SW: Apoptosis: a link between cancer genetics and chemotherapy. Cell 108: 153-164, 2002.

8. Eyler CE and Rich JN: Survival of the fittest: cancer stem cells in therapeutic resistance and angiogenesis. J Clin Oncol 26 $2839-2845,2008$

9. Hanahan D and Coussens LM: Accessories to the crime: functions of cells recruited to the tumor microenvironment Cancer Cell 21: 309-322, 2012.

10. Condeelis J and Pollard JW: Macrophages: obligate partners for tumor cell migration, invasion, and metastasis. Cell 124: 263-266, 2006.

11. Vermeulen L, Felipe De Sousa EM, van der Heijden M, et al: Wnt activity defines colon cancer stem cells and is regulated by the microenvironment. Nat Cell Biol 12: 468-476, 2010.

12. Wu A, Wei J, Kong L-Y, et al: Glioma cancer stem cells induce immunosuppressive macrophages/microglia. Neuro Oncol 12 1113-1125, 2010.

13. Sica A and Mantovani A: Macrophage plasticity and polarization: in vivo veritas. J Clin Invest 122: 787-795, 2012.

14. Murray PJ and Wynn TA: Protective and pathogenic functions of macrophage subsets. Nat Rev Immunol 11: 723-737, 2011.

15. Friedl $P$ and Wolf K: Tumour-cell invasion and migration: diversity and escape mechanisms. Nat Rev Cancer 3: 362-374, 2003.

16. Coffelt SB, Hughes R and Lewis CE: Tumor-associated macrophages: effectors of angiogenesis and tumor progression. Biochim Biophys Acta 1796: 11-18, 2009.

17. Beyer $M$ and Schultze JL: Regulatory $T$ cells in cancer 108: 804-811, 2006

18. Siveen KS and Kuttan G: Role of macrophages in tumour progression. Immunol Lett 123: 97-102, 2009.

19. Lewis CE and Pollard JW: Distinct role of macrophages in different tumor microenvironments. Cancer Res 66: 605-612, 2006.

20. $\mathrm{Hu} \mathrm{L}, \mathrm{McArthur} \mathrm{C}$ and Jaffe R: Ovarian cancer stem-like side-population cells are tumourigenic and chemoresistant. Br J Cancer 102: 1276-1283, 2010.

21. Spira AI, Marx PA, Patterson BK, Mahoney J, Koup RA, Wolinsky SM and Ho DD: Cellular targets of infection and route of viral dissemination after an intravaginal inoculation of simian immunodeficiency virus into rhesus macaques. J Exp Med 183: 215-225, 1996.
22. Tacke RS, Tosello-Trampont A, Nguyen V, Mullins DW and Hahn YS: Extracellular hepatitis $\mathrm{C}$ virus core protein activates STAT3 in human monocytes/macrophages/dendritic cells via an IL-6 autocrine pathway. J Biol Chem 286: 10847-10855, 2011.

23. Barboni B, Curini V, Russo V, et al: Indirect co-culture with tendons or tenocytes can program amniotic epithelial cells towards stepwise tenogenic differentiation. PLoS One 7: e30974, 2012.

24. Ohtaki H, Ylostalo JH, Foraker JE, et al: Stem/progenitor cells from bone marrow decrease neuronal death in global ischemia by modulation of inflammatory/immune responses. Proc Natl Acad Sci USA 105: 14638-14643, 2008.

25. Schmittgen TD and Livak KJ: Analyzing real-time PCR data by the comparative CT method. Nat Protoc 3: 1101-1108, 2008.

26. Subbaramaiah K, Morris PG, Zhou XK, et al: Increased levels of COX-2 and prostaglandin E2 contribute to elevated aromatase expression in inflamed breast tissue of obese women. Cancer Discov 2: 356-365, 2012.

27. Parazzoli S, Harmon J, Vallerie SN, Zhang T, Zhou H and Robertson RP: COX-2, not mPGES-1, is the dominant regulator of IL-1 $\beta$-induced PGE2 production and subsequent inhibition of pancreatic islet $\beta$-cell function during inflammation. J Biol Chem 2: 486-495, 2012.

28. Allavena P, Sica A, Solinas G, Porta C and Mantovani A: The inflammatory micro-environment in tumor progression: the role of tumor-associated macrophages. Crit Rev Oncol Hematol 66: 1-9, 2008.

29. Kim J and Hematti P: Mesenchymal stem cell-educated macrophages: A novel type of alternatively activated macrophages. Exp Hematol 37: 1445-1453, 2009.

30. Zhang QZ, Su WR, Shi SH, et al: Human gingiva-derived mesenchymal stem cells elicit polarization of M2 macrophages and enhance cutaneous wound healing. Stem Cells 28: 1856-1868, 2010.

31. Bouhlel M, Derudas B, Rigamonti E, et al: PPAR $\gamma$ activation primes human monocytes into alternative M2 macrophages with anti-inflammatory properties. Cell Metab 6: 137-143, 2007.

32. Monti P, Leone BE, Marchesi F, et al: The CC chemokine MCP-1/CCL2 in pancreatic cancer progression regulation of expression and potential mechanisms of antimalignant activity. Cancer Res 63: 7451-7461, 2003.

33. Singh B, Berry JA, Shoher A, Ramakrishnan V and Lucci A: COX-2 overexpression increases motility and invasion of breast cancer cells. Int J Oncol 26: 1393-1399, 2005.

34. Brown JR and DuBois RN: COX-2: a molecular target for colorectal cancer prevention. J Clin Oncol 23: 2840-2855, 2005.

35. Ferrandina G, Lauriola L, Zannoni G, et al: Increased cyclooxygenase-2 (COX-2) expression is associated with chemotherapy resistance and outcome in ovarian cancer patients. Ann Oncol 13: 1205-1211, 2002.

36. Roca H, Varsos ZS, Sud S, Craig MJ, Ying C and Pienta KJ: CCL2 and interleukin-6 promote survival of human CD11b+ peripheral blood mononuclear cells and induce M2-type macrophage polarization. J Biol Chem 284: 34342-34354, 2009.

37. Martinez FO, Gordon S, Locati $M$ and Mantovani A: Transcriptional profiling of the human monocyte-to-macrophage differentiation and polarization: New molecules and patterns of gene expression. J Immunol 177: 7303-7311, 2006.

38. Ding Y-B, Shi R-H, Tong J-D, et al: PGE2 up-regulates vascular endothelial growth factor expression in MKN28 gastric cancer cells via epidermal growth factor receptor signaling system. Exp Oncol 27: 108-113, 2005.

39. Dorsam RT and Gutkind JS: G-protein-coupled receptors and cancer. Nat Rev Cancer 7: 79-94, 2007.

40. Sica A, Schioppa T, Mantovani A and Allavena P: Tumour-associated macrophages are a distinct M2 polarised population promoting tumour progression: potential targets of anti-cancer therapy. Eur J Cancer 42: 717-727, 2006.

41. Aron-Wisnewsky J, Tordjman J, Poitou C, et al: Human adipose tissue macrophages: $\mathrm{m} 1$ and $\mathrm{m} 2$ cell surface markers in subcutaneous and omental depots and after weight loss. J Clin Endocrinol Metab 94: 4619-4623, 2009.

42. Hagemann T, Wilson J, Burke F, et al: Ovarian cancer cells polarize macrophages toward a tumor-associated phenotype. J Immunol 176: 5023-5032, 2006.

43. Zhu W, Huang L, Li Y, et al: Mesenchymal stem cell-secreted soluble signaling molecules potentiate tumor growth. Cell Cycle 10: 3198-3207, 2011.

44. Saraiva M and O'Garra A: The regulation of IL-10 production by immune cells. Nature Reviews Immunol 10: 170-181, 2010. 
45. Sinha P, Clements VK, Bunt SK, Albelda SM and Ostrand-Rosenberg S: Cross-talk between myeloid-derived suppressor cells and macrophages subverts tumor immunity toward a type 2 response. J Immunol 179: 977-983, 2007.

46. Wojnarowicz P, Gambaro K, de Ladurantaye M, et al: Overexpressing the CCL2 chemokine in an epithelial ovarian cancer cell line results in latency of in vivo tumourigenicity. Oncogenesis 1: 27-36, 2012.

47. Zhang J, Patel L and Pienta KJ: CC chemokine ligand 2 (CCL2) promotes prostate cancer tumorigenesis and metastasis. Cytokine Growth Factor Rev 21: 41-48, 2010.

48. Fujimoto H, Sangai T, Ishii G, et al: Stromal MCP-1 in mammary tumors induces tumor-associated macrophage infiltration and contributes to tumor progression. Int J Cancer 125: 1276-1284, 2009.

49. Ueno T, Toi M, Saji H, et al: Significance of macrophage chemoattractant protein-1 in macrophage recruitment, angiogenesis, and survival in human breast cancer. Clin Cancer Res 6 : 3282-3289, 2000.

50. Greenhough A, Smartt HJ, Moore AE, et al: The COX-2/PGE2 pathway: key roles in the hallmarks of cancer and adaptation to the tumour microenvironment. Carcinogenesis 30: 377-386, 2009.
51. Nakanishi Y, Nakatsuji M, Seno H, et al: COX-2 inhibition alters the phenotype of tumor-associated macrophages from M2 to M1 in ApcMin/+ mouse polyps. Carcinogenesis 32: 1333-1339, 2011

52. Whiteside TL: Regulatory T cell subsets in human cancer: are they regulating for or against tumor progression? Cancer Immunol Immunother 63: 67-72, 2014.

53. Eruslanov E, Daurkin I, Ortiz J, Vieweg J and Kusmartsev S: Pivotal Advance: Tumor-mediated induction of myeloid-derived suppressor cells and M2-polarized macrophages by altering intracellular PGE2 catabolism in myeloid cells. J Leukoc Biol 88: 839-848, 2010.

54. Heusinkveld M, van Steenwijk PJdV, Goedemans R, et al: M2 macrophages induced by prostaglandin E2 and IL-6 from cervical carcinoma are switched to activated M1 macrophages by CD4+ Th1 cells. J Immunol 187: 1157-1165, 2011.

55. Zhang W, Xu W and Xiong S: Macrophage differentiation and polarization via phosphatidylinositol 3-kinase/Akt-ERK signaling pathway conferred by serum amyloid $\mathrm{P}$ component. J Immunol 187: 1764-1777, 2011.

56. Lawrence $\mathrm{T}$ and Natoli G: Transcriptional regulation of macrophage polarization: enabling diversity with identity. Nat Rev Immunol 11: 750-761, 2011. 